\title{
Caracterização da macrofauna edáfica em sistemas de produção de grãos no Sudoeste do Piauí
}

\author{
Djavan Pinheiro Santos( ${ }^{(1)}$, Glenio Guimarães Santos ${ }^{(2)}$, Isis Lima dos Santos ${ }^{(3)}$, \\ Thiago Rodrigo Schossler ${ }^{(4)}$, Cíntia Carla Niva ${ }^{(5)}$ e Robélio Leandro Marchão(5)
}

\begin{abstract}
(1)Universidade Federal do Piauí, Campus Professora Cinobelina Elvas, Rodovia BR 135, Km 03, Caixa Postal 58, CEP 64900-000 Bom Jesus, PI, Brasil. E-mail: djavansantos@hotmail.com.br (2)Universidade Federal de Goiás, Campus Samambaia, Rodovia Goiânia/ Nova Veneza, Km 0, Caixa Postal 131, CEP 74690-900 Goiânia, GO, Brasil. E-mail: gleniogm@gmail.com (3)Universidade de Brasília,

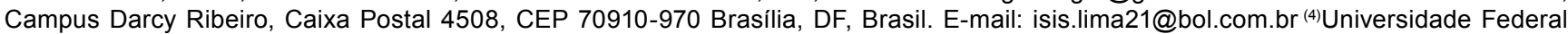
Rural de Pernambuco, Rua Dom Manoel de Medeiros, s/no, Dois Irmãos, CEP 52171-900 Recife, PE, Brasil. E-mail: schossler@msn.com (5)Embrapa Cerrados, Rodovia BR-020, Km 18, Caixa Postal 08223, CEP 73310-970 Planaltina, DF, Brasil. E-mail: cintia.niva@embrapa.br, robelio.marchao@embrapa.br
\end{abstract}

Resumo - O objetivo deste trabalho foi caracterizar a macrofauna edáfica em sistemas de produção de grãos sob plantio direto e convencional no Sudoeste do Piauí, na região de Matopiba. Amostras de solo foram coletadas nas camadas de $0,0-0,1,0,1-0,2$ e $0,2-0,3 \mathrm{~m}$, com serrapilheira, em áreas cultivadas predominantemente com milho e soja, sob preparo convencional e plantio direto, em solos com diferentes texturas. Remanescentes de vegetação nativa adjacentes foram amostrados como referência. A macrofauna edáfica nos diferentes sistemas de uso do solo foi identificada até o nível de famílias, as quais foram agrupadas de acordo com suas funções ecológicas. Os grupos mais abundantes nas áreas amostradas foram Isoptera, Hymenoptera e Coleoptera, este com o maior número de famílias. A análise de componentes principais revelou que os grupos funcionais geófago/bioturbador, fitófago/praga e predador/parasita estão associados a solos de textura argilo-siltosa; enquanto o grupo detritívoro/decompositor, a solos mais arenosos. Os sistemas de manejo do solo alteram a estrutura da comunidade de macroinvertebrados edáficos em relação à condição natural de Cerrado. O plantio direto proporciona maior abundância e riqueza de espécies que o preparo convencional, e minimiza o impacto de sistemas agrícolas sobre a biodiversidade da macrofauna edáfica.

Termos para indexação: bioindicadores, biodiversidade do solo, Cerrado, grupo funcional, invertebrados do solo, qualidade do solo.

\section{Characterization of soil macrofauna in grain production systems in the Southeastern state of Piauí, Brazil}

\begin{abstract}
The objective of this work was to characterize the edaphic macrofauna in agricultural grain production systems under no-tillage and conventional tillage, in the Shoutheastern state of Piauí, in the Matopiba region, Brazil. Soil samples were taken from the $0.0-0.1,0.1-0.2$, and $0.2-0.3$-m layers, with litter, in areas predominantly cropped with corn and soybean, under conventional and no-tillage systems, in soils with different textures. Fragments of native vegetation in adjacent areas were used as a reference. The edaphic macrofauna in the different land uses was identified down to family level, and the families were grouped according to their ecological functions. The most abundant groups in the sampled areas were Isoptera, Hymenoptera, and Coleoptera, the latter with the highest number of families. Principal component analysis revealed that the functional groups geophages/bioturbators, phytophages/pests, and predators/parasites were associated with clayey-silty soils, and detritivores/decomposers with sandy soils. Soil management systems change the community structure of edaphic macroinvertebrates in comparison with the natural Cerrado condition. No-tillage system promotes greater abundance and species richness than conventional tillage, and minimizes the impact of agricultural systems on edaphic macrofauna biodiversity.
\end{abstract}

Index terms: bioindicators, soil biodiversity, Cerrado, functional group, soil invertebrates, soil quality.

\section{Introdução}

O Sudoeste piauiense pertence à região denominada de Matopiba, em referência aos estados do Maranhão,
Tocantins, Piauí e Bahia, e é a principal fronteira agrícola remanescente no bioma Cerrado, onde se estima que a área de lavouras cresça 5,3 milhões

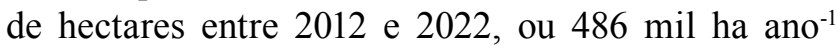

Pesq. agropec. bras., Brasília, v.51, n.9, p.1466-1475, set. 2016

DOI: $10.1590 / \mathrm{S} 0100-204 X 2016000900045$ 
(Mingoti et al., 2014). Nas últimas três décadas, tem-se observado expressivo aumento da atividade agropecuária nessa região do Cerrado, com intensa substituição da vegetação nativa por áreas cultivadas, especialmente para produção de alimentos, fibra e energia (Acompanhamento..., 2015).

O manejo inadequado de solos de textura média ou arenosa, predominantes na região, tem deteriorado sua estrutura, notadamente sob preparo convencional (Marchão et al., 2008). Essa degradação está relacionada a alterações nas propriedades físicas, químicas $\mathrm{e}$ biológicas dos solos, causadas pelo preparo excessivo, assim como pela ausência de uma cobertura morta efetiva. Em trabalho na região, Pragana et al. (2012) relataram que a substituição da vegetação nativa por sistemas agrícolas em monocultivo causa mudanças abruptas no ecossistema, com prejuízo para os serviços ecossistêmicos da paisagem e para a qualidade do solo. Segundo os autores, a adoção de sistemas conservacionistas, como o sistema plantio direto (SPD) baseado na rotação de culturas, pode ser uma alternativa para evitar perdas de solo, água, nutrientes e da biodiversidade. Os solos cultivados sob SPD podem abrigar comunidades complexas de macroinvertebrados, que estimulam os processos de decomposição da matéria orgânica (Hendrix et al., 1986), em comparação a sistemas convencionais com monocultivo.

A macrofauna edáfica refere-se a invertebrados de solo com dimensão corporal superior a $2 \mathrm{~mm}$ (Swift et al., 1979). Ela pode ser influenciada tanto pela quantidade quanto pela qualidade de material vegetal aportado ao solo (Carrillo et al., 2011), e seus indivíduos podem ser classificados de acordo com seu papel funcional no solo, como os grupos transformadores da serrapilheira e os engenheiros do ecossistema, que contribuem com serviços ecossistêmicos de suporte e regulação (Brown et al., 2015).

Espécies, famílias ou grupos funcionais podem servir como bioindicadores de mudanças ambientais. Os invertebrados de solo mais utilizados como bioindicadores pertencem à classe Hymenoptera (formigas e abelhas), Isoptera (cupins), Coleoptera (besouros), Arachnida (aranhas) e Oligochaeta (minhocas) (Wink et al., 2005). Eles podem ser usados para avaliar a biodiversidade, a atividade biológica do solo e a sustentabilidade dos sistemas de manejo (Lavelle et al., 2006). No entanto, apesar da importância dos invertebrados para a manutenção da qualidade do solo, ainda se conhece pouco sobre a macrofauna edáfica em áreas antropizadas (Brown et al., 2015). No bioma Cerrado, o conhecimento sobre a macrofauna edáfica e o impacto de atividades agropecuárias sobre ela ainda é restrito a áreas e sistemas de produção nas partes central e sul do bioma (Benito et al., 2005; Silva et al., 2006; Santos et al., 2008; Marchão et al., 2009; Portilho et al., 2011). No Cerrado de Matopiba, as informações restringem-se ao Oeste Baiano (Marchão et al., 2008). Esses trabalhos demonstram que sistemas mais conservacionistas, que proporcionam maior cobertura do solo e diversidade de resíduos vegetais aportados ao solo, favorecem grupos específicos da macrofauna, ainda que o impacto antrópico do manejo do solo normalmente resulte em perdas na densidade de organismos em comparação aos ecossistemas naturais.

No Piauí, existem informações em regiões de transição com outros biomas, mas não existem estudos na região de Cerrado típico, onde predomina agricultura intensiva. Alguns trabalhos tratam da fauna epigeica, com foco na serrapilheira de áreas de pastagens (Nunes et al., 2012; Luz et al., 2013), de cultivos de cana-deaçúcar (Abreu et al., 2014), ou ainda sobre a macrofauna em sistemas agroflorestais (Lima et al., 2010). Com a rápida transformação de sistemas naturais em sistemas agrícolas na região, a macrofauna edáfica pode sofrer perdas em sua abundância e diversidade. Em solos tropicais, que são mais impactados pela intensificação da agricultura, a perda na biodiversidade, antes mesmo de sua caracterização, é uma possibilidade preocupante (Giller et al., 1997).

O objetivo deste trabalho foi caracterizar a macrofauna edáfica em sistemas de produção de grãos sob plantio direto e convencional, em fazendas representativas da região Matopiba.

\section{Material e Métodos}

O estudo foi realizado na região do Cerrado de Matopiba, em fazendas representativas de sistemas de produção de grãos, em quatro municípios no Sudoeste do estado do Piauí: Baixa Grande do Ribeira $\left(07^{\circ} 48^{\prime} 10^{\prime \prime}\right.$ $\mathrm{S}$ e $45^{\circ} 00^{\prime} 60^{\prime \prime} \mathrm{W}$, altitude de $\left.600 \mathrm{~m}\right)$, Sebastião Leal $\left(07^{\circ} 39^{\prime} 14^{\prime \prime} \mathrm{S}\right.$ e $44^{\circ} 02^{\prime} 37^{\prime \prime} \mathrm{W}$, altitude de $\left.450 \mathrm{~m}\right)$, Uruçuí $\left(08^{\circ} 14^{\prime} 07^{\prime \prime} \mathrm{S}\right.$ e $44^{\circ} 38^{\prime} 09^{\prime \prime} \mathrm{W}$, altitude de $\left.550 \mathrm{~m}\right)$ e Bom Jesus (09 $19^{\circ} 35^{\prime \prime} \mathrm{S}$ e $44^{\circ} 50^{\prime} 36^{\prime \prime} \mathrm{W}$, altitude de $\left.600 \mathrm{~m}\right)$.

O clima da região é Aw tropical de savana, conforme classificação de Köppen, com predominância da 
estação chuvosa entre os meses de outubro a abril e precipitação média anual de $1.200 \mathrm{~mm}$. Predominam, na região, solos da classe Latossolo Amarelo distrófico típico, textura franco-argilo-arenosa, com vegetação remanescente de cerrado subcaducifólio (Pragana et al., 2012). A análise da distribuição granulométrica dos solos foi realizada conforme Claessen (1997) (Tabela 1). A seleção das áreas e fazendas foi feita

Tabela 1. Granulometria do solo na camada de $0-0,3 \mathrm{~m}$ nas áreas amostradas no Sudoeste do estado do Piauí, na região de Matopiba.

\begin{tabular}{lccccc}
\hline Município & Argila & Silte & $\begin{array}{c}\text { Areia } \\
\text { fina }\end{array}$ & $\begin{array}{c}\text { Areia } \\
\text { grossa }\end{array}$ & $\begin{array}{c}\text { Areia } \\
\text { total }\end{array}$ \\
\hline Baixa Grande do Ribeira & --------------- \\
$\left(\mathrm{g} \mathrm{kg}^{-1}\right)$ & --------------- \\
Bom Jesus & 218 & 125 & 70 & 187 & 257 \\
Sebastião Leal & 319 & 67 & 108 & 643 & 751 \\
Uruçuí & 187 & 24 & 147 & 470 & 615 \\
\hline
\end{tabular}

com vistas à contemplação de áreas com diferentes texturas. Os históricos das glebas nas propriedades selecionadas estão descritos na Tabela 2 .

A amostragem da macrofauna foi realizada em abril de 2012, em áreas de produção de grãos sob plantio convencional (PC) e plantio direto (PD), bem como em remanescentes da vegetação nativa de cerrado $(\mathrm{VN})$, adjacentes às áreas de cultivo. Em cada gleba, os pontos de coleta foram definidos após demarcação com GPS de 25 pontos equidistantes, em uma malha regular, em parcela com 1,0 ha (100x100 m). Cinco pontos, sorteados entre os 25 demarcados, foram utilizados para compor as repetições em cada área. Os monólitos foram coletados em uma pequena trincheira, na camada de $0-0,3 \mathrm{~m}$, posicionada ao redor de cada um dos cinco pontos. O local de abertura da trincheira foi definido após lançamento aleatório de quadro metálico amostrador, com $25 \times 25 \mathrm{~cm}$, segundo recomendações do TSBF (Tropical Soil Biology and Fertility Program),

Tabela 2. Histórico dos diferentes sistemas de manejo do solo nos municípios amostrados no Sudoeste do estado do Piauí, na região de Matopiba.

\begin{tabular}{ll}
\hline Sistema de manejo & \multicolumn{1}{c}{ Descrição } \\
\hline Plantio convencional & $\begin{array}{l}\text { Abertura em } 1990 \text { e conduzida sob preparo convencional até 1994/95 (ano de aplicação de calagem) e cultivo de arroz, ficando } \\
\text { em pousio até 1999. Na safra de 2000/01 a 2009/10, preparo convencional e cultivo de soja, seguido de soja/milheto. Dezesseis } \\
\text { anos de sua implementação desde a abertura da área. }\end{array}$ \\
Plantio direto & $\begin{array}{l}\text { Abertura em 1990. Quatro anos conduzida segundo o preparo convencional, ficando em pousio de 1995 a 2006. Aplicação de } \\
\text { calagem na safra de 2006/07 e a partir desse ano, cultivo com soja/milheto em todos os anos. Seis anos de implementação do } \\
\text { plantio direto. }\end{array}$ \\
\hline
\end{tabular}

\section{Sebastião Leal}

Plantio convencional Abertura em 2010 e conduzida sob preparo convencional com aplicação de calagem e cultivo de milheto na safra de $2010 / 11$. Preparo convencional e cultivo de soja na safra de 2011/12. Dois anos de sua implementação desde a abertura da área.

Plantio direto

Abertura em 1997, conduzida sob preparo convencional e aplicação de calagem. Nos anos de 1998 a 2000 , cultivo da soja. Na safra de 2000/01 cultivo do milheto. Do ano de 2001 até a safra de 2007/2008, cultivo da soja, seguido de algodão na 2008/09, milho na safra de 2009/10 e soja até a safra de 2011/12 com quatorze anos de implementação do plantio direto.

\section{Uruçuí}

Plantio convencional

Abertura em 2009, conduzida sob preparo convencional e aplicação de calagem e cultivo de arroz na safra 2009/10. Preparo convencional e aplicação de calagem e cultivo de soja/milheto em 2010/11. Aplicação de calagem e cultivo da soja na safra de 2011/2012 e três anos de implantação desde a abertura da área.

Abertura em 1999, conduzida sob preparo convencional e aplicação de calagem e cultivo de arroz nas safras 2000/01 e 2001/02,

Plantio direto cultivo da soja na safra de 2002/03 a 2006/07, aplicação de calagem na safra de 2009/10 e cultivo da soja. Na safra de 2007/08 e 2008/09 cultivo de soja/milheto. Na safra de 2010/11, cultivo do milho e na safra de 2011/12, cultivo de soja/feijão e treze anos de implementação do plantio direto.

Bom Jesus

Abertura em 2009, conduzida sob preparo convencional, aplicação de calagem e cultivo do feijão na safra de 2010/11. Preparo convencional e aplicação de calagem com cultivo de feijão na safra de 2011/12 e dois anos de implementação desde a abertura da área.

Plantio convencional 
conforme Anderson \& Ingram (1993). No momento da amostragem, os monólitos foram divididos nas camadas 0-0,1, 0,1-0,2 e 0,2-0,3 m, e incluíram a serrapilheira. Após a triagem manual, realizada a céu aberto nas sedes das fazendas, os invertebrados coletados foram armazenados em potes com álcool diluído, na proporção de $70 \%$, e posteriormente encaminhados ao laboratório de entomologia da Universidade Federal do Piauí, em Bom Jesus, PI, para contagem e identificação dos organismos até o nível taxonômico de famílias.

Os resultados de abundância absoluta para cada família, em cada área, foram expressos em densidade relativa $\left(\mathrm{n}^{\mathrm{o}}\right.$ de indivíduos por $\mathrm{m}^{2}$ ) na camada de 0-0,3 m, incluso a serrapilheira. A densidade relativa para os usos PC, PD e VN foi calculada a partir da média das áreas por fazenda. A distribuição vertical em cada área foi calculada a partir das médias das cinco repetições em cada camada. Os dados de densidade foram submetidos ao teste de Shapiro Wilk e, mesmo após transformação em raiz de $\mathrm{x}+1$, verificou-se ausência de normalidade. O erro-padrão das médias foi apresentado como medida de dispersão dos dados.

Com o propósito de verificar as relações entre os grupos funcionais da macrofauna e a textura do solo, em cada uma das áreas amostradas, realizou-se a análise de componentes principais (ACP) em matriz composta por 36 linhas (três áreas/sistemas, quatro fazendas e três camadas) e quatro colunas (grupos funcionais), com vistas à identificação das variáveis dos grupos funcionais que contribuíram com maior peso, na combinação linear dos dois primeiros componentes principais. Para auxiliar na interpretação dos resultados, as famílias foram agrupadas de acordo com suas funções ecológicas, conforme classificação de Brown et al. (2015), em que a fauna edáfica é dividida em quatro grupos funcionais: geófago/bioturbador; detritívoro/ decompositor; fitófago/praga e predador/parasita. A ACP foi realizada com o programa estatístico Canoco, versão 4.5 (Food and Agriculture Organization of the United Nations, Nova Iorque, EUA), em que os dados de textura do solo foram plotados como variáveis explicativas, mas não foram incluídos na análise como variável resposta no modelo estatístico.

\section{Resultados e Discussão}

A soma da densidade total relativa da macrofauna edáfica quantificada nas três áreas foi de 2.121 indivíduos por $\mathrm{m}^{2}$, distribuídos em quatro classes,
14 ordens e 32 famílias (Tabela 3). Os grupos taxonômicos mais abundantes, em ordem decrescente de densidade relativa, foram: Isoptera $(66,86 \%)$, Hymenoptera (15,79\%), Coleoptera larva $(6,84 \%)$, Coleoptera adulto $(6,27 \%)$, Araneae $(1,51 \%)$, Hemiptera $(0,75 \%)$, Haplotaxida $(0,42 \%)$, Lepidoptera $(0,38 \%)$, Scolopendromorpha $(0,33 \%)$, Blattodea $(0,28 \%)$, Diptera $(0,19 \%)$, Scorpiones $(0,14 \%)$, Dermaptera $(0,14 \%)$ e Orthoptera $(0,09 \%)$.

Na comparação com outros trabalhos em áreas com cultivo de grãos no Cerrado, os valores de densidade nas áreas de cultivo do presente trabalho foram mais baixos (Tabela 4), mas com a mesma tendência de maior densidade no plantio direto, em comparação ao preparo convencional do solo (Silva et al., 2006; Marchão et al., 2009; Padoa, 2010). Essa menor densidade pode estar relacionada às altas temperaturas observadas nesta região do Cerrado. Por exemplo, no município de Bom Jesus, a temperatura máxima média no período de $1971-2010$ atingiu $36^{\circ} \mathrm{C}$. Em regiões tropicais, a macrofauna do solo deve ser amostrada durante a estação chuvosa (Lavelle, 1988), quando os fatores climáticos de temperatura e umidade ainda não são limitantes. Ainda assim, a temperatura e umidade são comumente apontados como determinantes para a dinâmica populacional da macrofauna do solo (Lavelle et al., 2006; Santos et al., 2008; Manhães et al., 2013).

A estrutura da comunidade de invertebrados diferiu entre as áreas e sistemas de manejos. As áreas cultivadas sob PC e PD apresentaram densidade média de 136 e 214 indivíduos $\mathrm{m}^{-2}$, respectivamente, e uma densidade média de 1.771 indivíduos $\mathrm{m}^{-2}$ nas áreas de vegetação nativa. As ordens Isoptera $(57,35 \%)$ e Coleoptera larva (18,38\%) e adulto $(7,35 \%)$ predominaram nas áreas sob PC, enquanto as ordens Coleoptera adulto $(51,4 \%)$ e larva $(23,83 \%)$, seguidas de Araneae (10,28\%), predominaram sob PD. Nas áreas de $\mathrm{VN}$, predominaram os grupos Isoptera $(75,55 \%) \mathrm{e}$ Hymenoptera $(18,46 \%)$. De acordo com a classificação de grupos funcionais (Brown et al., 2015), os grupos dominantes identificados nas áreas de vegetação nativa são geófagos/bioturbadores (Isoptera e Hymenoptera), o que demonstra a importância de "engenheiros do ecossistema" em áreas nativas (Lavelle et al., 2014).

Os indivíduos do grupo Isoptera (cupins) representaram $66,86 \%$ da macrofauna edáfica quantificada nas três áreas, semelhantemente ao resultado de outros trabalhos em Latossolos do 
Cerrado, em que essa ordem também predominou em relação às demais (Benito et al., 2005; Silva et al., 2006; Marchão et al., 2008). Esse táxon, bastante comum no Cerrado, ocorreu em todos os sistemas de manejo, mas sua densidade relativa foi maior nas áreas cultivadas, $o$ que também está de acordo com a literatura no Cerrado (Constantino, 2005; Marchão et al., 2008, 2009). A família Termitidae foi altamente predominante sob PC e VN (Tabela 3). A grande abundância desta família sob PC demonstra a resiliência de cupins ao revolvimento e exposição do solo. Oliveira et al. (2011) relataram que as subfamílias Nasutitermitinae e Termitinae são as mais abundantes em estudo no Cerrado, em que 12 morfoespécies foram identificadas dentro de

Tabela 3. Média \pm erro-padrão da densidade relativa (indivíduos por $\left.\mathrm{m}^{2}\right)^{(1)}$ e da riqueza de famílias da macrofauna edáfica em áreas agrícolas sob plantio convencional (PC) e plantio direto (PD), e em vegetação nativa de cerrado (VN), no Sudoeste do estado do Piauí, na região de Matopiba.

\begin{tabular}{|c|c|c|c|c|c|}
\hline \multirow[t]{2}{*}{ Classe } & \multirow[t]{2}{*}{ Ordem } & \multirow[t]{2}{*}{ Família } & \multicolumn{3}{|c|}{ Número de indivíduos por $\mathrm{m}^{2}$} \\
\hline & & & Plantio convencional & Plantio direto & Vegetação nativa \\
\hline Arachnida & Araneae & Theridiidae & $4 \pm 2,01$ & $21 \pm 12,56$ & $6 \pm 2,26$ \\
\hline Arachnida & Araneae & Licosidae & 0 & $1 \pm 0,80$ & 0 \\
\hline Arachnida & Scorpiones & Scorpionidae & 0 & $1 \pm 0,80$ & $2 \pm 1,53$ \\
\hline Insecta & Coleoptera Ad & Carabidae & $3 \pm 1,31$ & $5 \pm 2,07$ & 0 \\
\hline Insecta & Coleoptera Ad & Scarabaeidae & $6 \pm 3,46$ & $86 \pm 61,27$ & $3 \pm 2,26$ \\
\hline Insecta & Coleoptera Ad & Nitidulidae & 0 & $2 \pm 2,40$ & 0 \\
\hline Insecta & Coleoptera Ad & Staphylinidae & 0 & $12 \pm 1,53$ & $5 \pm 3,81$ \\
\hline Insecta & Coleoptera Ad & Languridae & 0 & $4 \pm 4,00$ & $2 \pm 1,60$ \\
\hline Insecta & Coleoptera Ad & Tenebrionidae & $1 \pm 0,80$ & $1 \pm 0,80$ & $2 \pm 2,40$ \\
\hline Insecta & Coleoptera Ad & Geotrupidae & 0 & 0 & $1 \pm 0,80$ \\
\hline Insecta & Coleoptera La & Elateridae & $18 \pm 14,58$ & $29 \pm 21,59$ & $25 \pm 10,15$ \\
\hline Insecta & Coleoptera La & Curculionidae & 0 & $1 \pm 0,80$ & $1 \pm 0,80$ \\
\hline Insecta & Coleoptera La & Melolontidae & $6 \pm 4,60$ & $8 \pm 4,23$ & $16 \pm 11,31$ \\
\hline Insecta & Coleoptera La & Chrysomelidae & $1 \pm 0,80$ & $13 \pm 5,39$ & $27 \pm 12,43$ \\
\hline Insecta & Hemiptera & Cicadidae & $2 \pm 1,60$ & 0 & $1 \pm 0,80$ \\
\hline Insecta & Hemiptera & Reduviidae & $1 \pm 0,80$ & 0 & 0 \\
\hline Insecta & Hemiptera & Pentatomidae & $1 \pm 0,80$ & $7 \pm 6,18$ & $2 \pm 1,53$ \\
\hline Insecta & Hemiptera & Cydinidae & 0 & 0 & $2 \pm 2,40$ \\
\hline Insecta & Hymenoptera & Formicidae & $2 \pm 1,53$ & $6 \pm 4,53$ & $327 \pm 299,59$ \\
\hline Insecta & Isoptera & Termitidae & $78 \pm 78,40$ & $2 \pm 1,60$ & $1331 \pm 982,42$ \\
\hline Insecta & Isoptera & Rhynotermitidae & 0 & 0 & $7 \pm 5,29$ \\
\hline Insecta & Blattodea & Blattellidae & 0 & 0 & $1 \pm 0,80$ \\
\hline Insecta & Blattodea & Blaberidae & $1 \pm 0,80$ & $2 \pm 2,40$ & $2 \pm 1,60$ \\
\hline Insecta & Lepidoptera & Nymphalidae & $1 \pm 0,80$ & $6 \pm 1,31$ & $1 \pm 0,80$ \\
\hline Insecta & Orthoptera & Grylidae & 0 & $1 \pm 0,80$ & 0 \\
\hline Insecta & Orthoptera & Acrididae & $1 \pm 0,80$ & 0 & 0 \\
\hline Insecta & Dermaptera & Anisolabididae & 0 & $1 \pm 0,80$ & 0 \\
\hline Insecta & Dermaptera & Labiduridae & $1 \pm 0,80$ & $1 \pm 0,80$ & 0 \\
\hline Insecta & Diptera & Muscidae & $1 \pm 0,80$ & $1 \pm 0,80$ & $1 \pm 0,80$ \\
\hline Insecta & Diptera & Chaoboridae & 0 & 0 & $1 \pm 0,80$ \\
\hline Chilopoda & Scolopendromorpha & Scolopocryptopidae & $1 \pm 0,80$ & $1 \pm 0,80$ & $5 \pm 1,60$ \\
\hline Oligochaeta & Haplotaxida & - & $7 \pm 4,60$ & $2 \pm 1,60$ & 0 \\
\hline Soma da densidade & & & $136 \pm 74,70$ & $214 \pm 58,40$ & $1771 \pm 909,03$ \\
\hline $\mathrm{N}^{\mathrm{o}}$ de famílias & & & $19 \pm 2,53$ & $24 \pm 0,65$ & $23 \pm 1,89$ \\
\hline
\end{tabular}

${ }^{(1)}$ Calculada a partir da média das áreas por fazenda. Ad e La referem-se às ordens Coleoptera adulto e Coleoptera larva, respectivamente. 
11 gêneros, a maioria humívora. Portilho et al. (2011) também relataram maiores densidades de Termitidae em sistemas de integração lavoura-pecuária, em Latossolo do Cerrado do Mato Grosso do Sul.

Sob PD, os invertebrados detritívoros/ decompositores (Coleoptera) foram predominantes, seguidos dos predadores (Arachnida), o que sugere alta atividade de decomposição e uma estrutura

Tabela 4. Levantamento da macrofauna edáfica em sistemas de produção de grãos em diferentes municípios na região do Cerrado.

\begin{tabular}{|c|c|c|c|}
\hline Sistema de manejo & $\begin{array}{c}\text { Densidade } \\
\left(\text { indivíduos por } \mathrm{m}^{2}\right)^{(1)}\end{array}$ & $\begin{array}{c}\mathrm{N}^{\mathrm{o}} \mathrm{de} \\
\text { grupos }^{(2)}\end{array}$ & Referências \\
\hline & \multicolumn{3}{|c|}{ Baixa Grande do Ribeira, PI } \\
\hline Plantio direto & 250 & 7 & \multirow[t]{2}{*}{ Este estudo } \\
\hline \multirow[t]{2}{*}{ Plantio convencional } & 106 & 7 & \\
\hline & \multicolumn{3}{|c|}{ Sebastião Leal, PI } \\
\hline Plantio direto & 115 & 7 & \multirow[t]{2}{*}{ Este estudo } \\
\hline \multirow[t]{2}{*}{ Plantio convencional } & 3 & 1 & \\
\hline & \multicolumn{3}{|c|}{ Uruçuí, PI } \\
\hline Plantio direto & 144 & 6 & \multirow[t]{2}{*}{ Este estudo } \\
\hline \multirow[t]{2}{*}{ Plantio convencional } & 80 & 9 & \\
\hline & \multicolumn{3}{|c|}{ Bom Jesus, PI } \\
\hline Plantio direto & 358 & 8 & \multirow[t]{2}{*}{ Este estudo } \\
\hline \multirow[t]{2}{*}{ Plantio convencional } & 349 & 6 & \\
\hline & \multicolumn{3}{|c|}{ Maracaju, MS } \\
\hline Plantio direto & 518 & 8 & \multirow{2}{*}{$\begin{array}{c}\text { Lourente et al. } \\
\text { (2007) }\end{array}$} \\
\hline \multirow[t]{2}{*}{ Plantio convencional } & 470 & 6 & \\
\hline & \multicolumn{3}{|c|}{ Dourados, MS } \\
\hline Plantio direto & 914 & 11 & \multirow{2}{*}{$\begin{array}{l}\text { Silva et al. } \\
\text { (2006) }\end{array}$} \\
\hline \multirow[t]{2}{*}{ Plantio convencional } & 485 & 11 & \\
\hline & \multicolumn{3}{|c|}{ Correntina, BA } \\
\hline Plantio direto & 479 & 6 & \multirow{2}{*}{$\begin{array}{l}\text { Marchão et al. } \\
\text { (2008) }\end{array}$} \\
\hline \multirow[t]{2}{*}{ Plantio convencional } & - & - & \\
\hline & \multicolumn{3}{|c|}{ Santo Antônio de Goiás, GO } \\
\hline Plantio direto & 424 & 9 & \multirow{2}{*}{$\begin{array}{l}\text { Santos et al. } \\
\text { (2008) }\end{array}$} \\
\hline \multirow[t]{2}{*}{ Plantio convencional } & - & - & \\
\hline & \multicolumn{3}{|c|}{ Planaltina, DF } \\
\hline Plantio direto & 2.053 & 13 & \multirow{2}{*}{$\begin{array}{l}\text { Marchão et al. } \\
\text { (2009) }\end{array}$} \\
\hline \multirow[t]{2}{*}{ Plantio convencional } & 996 & 12 & \\
\hline & Un & aí, MG & \multirow{3}{*}{ Padoa (2010) } \\
\hline Plantio direto & 300 & 10 & \\
\hline Plantio convencional & 147 & 5 & \\
\hline
\end{tabular}

${ }^{(1)}$ Nos casos com mais de um sistema de manejo, os dados de densidade foram obtidos a partir da média. ${ }^{(2)}$ Referente aos níveis de ordem, família, subfamília ou gênero, tendo-se tomado o maior valor observado, nos casos com mais de um sistema de manejo. trófica mais equilibrada nesse sistema. Levando-se em conta os adultos e as larvas, a ordem Coleoptera foi a mais diversa no PD, onde ocorreram 10 famílias (Tabela 3). Embora algumas famílias atuem como pragas na agricultura, a maioria das espécies de Coleoptera é benéfica para o solo, pela construção de galerias que favorecem a infiltração da água e por sua ação incorporadora de matéria orgânica ao solo (Correia \& Oliveira, 2005). As famílias Scarabaeidae $(40,19 \%)$, na forma de adulto, seguida de Elateridae $(13,55 \%)$ e Chrysomelidae $(6,07 \%)$, na forma de larva, predominaram. Os indivíduos dessas famílias apresentam hábito alimentar diversificado (Hanski \& Cambefort, 1991), o que sugere que a rotação de culturas e a preservação da estrutura do solo sob PD proporciona uma condição mais favorável à colonização por essas famílias.

Deve-se ressaltar que o PD possibilitou maior ocorrência de indivíduos dos grupos Staphylinidae, indício de que proporciona um ambiente mais equilibrado que favorece o desenvolvimento de grupos predadores em razão da maior oferta de alimentos e de microclima favorável (House \& Parmelee, 1985). Muitas espécies dessas famílias são comumente encontradas em áreas sob culturas agrícolas e, por sua natureza predatória, elas destacam-se como agentes de controle biológico de pragas (Sotherton, 1984). Além de predadores, os representantes da família Staphylinidae são também detritívoros/decompositores, e podem ser coprófagos, necrófagos ou consumidores de fungos e materiais em decomposição (Brown et al., 2015). Portilho et al. (2011) apontaram os Staphylinidae como potenciais bioindicadores da qualidade do solo, pois observaram maior abundância desse grupo em área de floresta semidecídua e em sistemas de produção conservacionistas. Segundo Hendrix et al. (1986), a adoção do sistema plantio direto favorece a ocorrência dessas famílias, que se alimentam, principalmente, de material vegetal ou animal em decomposição.

As famílias Melolontidae e Chrysomelidae, da ordem Coleoptera, apresentaram menores densidades relativas na forma larval, sob o cultivo, em comparação à VN (Tabela 3), possivelmente, pelo fato de esses organismos serem sensíveis às perturbações antrópicas. Muitas espécies dessas famílias apresentam hábito alimentar diversificado e desempenham diferentes funções no solo, como decomposição e incorporação de material orgânico, mineralização de nutrientes, aeração e agregação do solo, além de servirem 
de alimento para diversos organismos predadores (Oliveira, 2005). Menores densidades de larvas de indivíduos da ordem Coleoptera nas áreas agrícolas também podem estar relacionadas ao uso mais intensivo do solo e à aplicação intensa de defensivos.

A família Formicidae (Hymenoptera), muito abundante em áreas de Cerrado, também apresentou menores densidades nas áreas agrícolas, em comparação à VN. Resultado similar foi reportado por Silva et al. (2006), em estudo de invertebrados do solo sob diferentes sistemas de produção, em Latossolo da região do Cerrado de Mato Grosso do Sul. Assim como os cupins, esse grupo é considerado importante para o funcionamento do solo, uma vez que sua presença está associada ao processo de decomposição de material orgânico e ao fato de que ele participa do equilíbrio dinâmico de sistemas agrícolas conservacionistas (Bruyn, 1999).

Apesar da predominância, nas áreas estudadas, de grupos considerados engenheiros do ecossistema, os Oligochaeta foram muito pouco abundantes nas áreas cultivadas, e ausentes na VN (Tabela 3). Esse resultado contrasta com relatos no Sul do país, onde a abundância e a riqueza de minhocas não diferem entre áreas de floresta e de plantio direto, e podem ser utilizadas como indicadora da qualidade do solo (Bartz et al., 2013). Em estudos em outras regiões de Cerrado, o grupo Oligochaeta pode figurar, até mesmo entre um dos quatro mais abundantes (Santos et al., 2008; Marchão et al., 2009; Vendrame et al., 2009). No Piauí, contudo, as minhocas ainda não haviam sido amostradas até 2007 (Brown et al., 2006). O fato de esse grupo ter sido amostrado apenas em PC e PD, no presente trabalho, sugere que as minhocas coletadas sejam, possivelmente, de espécies exóticas. Entretanto, mais estudos são necessários para conhecer a diversidade, ecologia e importância dos Oligochaeta neste ambiente, principalmente como indicadores da qualidade do solo em sistemas naturais ou em agroecossistemas no bioma Cerrado.

$\mathrm{Na}$ avaliação da distribuição vertical da macrofauna edáfica (Figura 1), verificou-se que as áreas sob PC e $\mathrm{VN}$ apresentaram maior proporção de indivíduos na camada $0-0,1 \mathrm{~m}$, que concentrou 78,57 e $63,83 \%$ da abundância, respectivamente. Por outro lado, nas áreas sob PD a colonização do perfil foi mais uniforme, com proporções semelhantes nas camadas $0-0,1$ e 0,1-0,2 m, com maior contribuição da serrapilheira. A camada de $0,2-0,3 \mathrm{~m}$ foi a que apresentou menor proporção, independentemente do tipo de uso da terra, o que está de acordo com outros trabalhos realizados em Latossolos de Cerrado, como os de Marchão et al. (2009) e Batista et al. (2014), que observaram maior
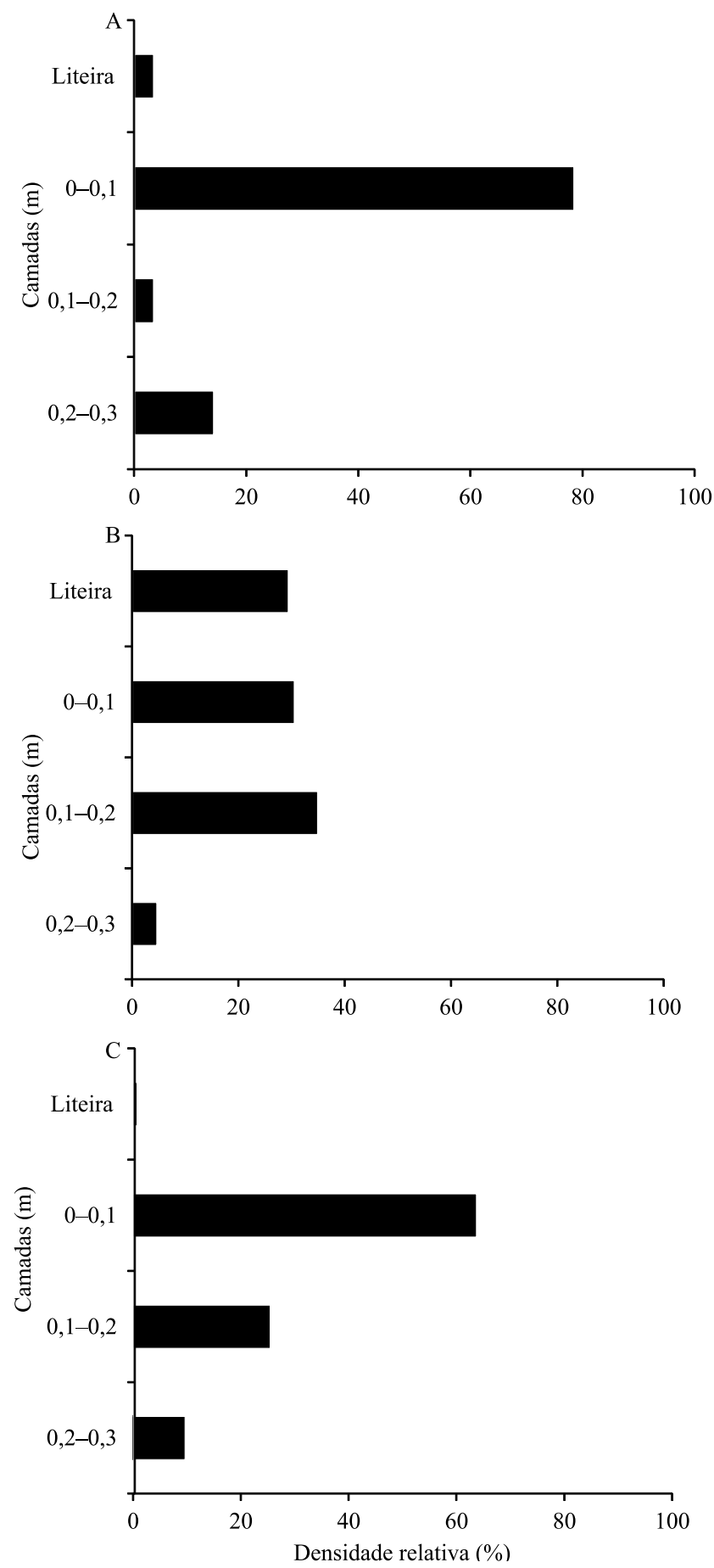

Figura 1. Densidade relativa da macrofauna edáfica conforme a profundidade dos solos sob: A, preparo convencional; B, plantio direto; e $\mathrm{C}$, vegetação nativa de cerrado. 
atividade da fauna edáfica nas camadas superficiais. Uma distribuição mais uniforme dos organismos no perfil do solo sob PD demonstra que este sistema proporciona condições mais favoráveis à colonização pela macrofauna, provavelmente em razão de uma maior mobilidade devida à escavação, construção e manutenção de galerias e bioporos.

As relações entre grupos funcionais da macrofauna edáfica com as frações granulométricas do solo, nas áreas amostradas, podem ser visualizadas nos resultados da análise de componentes principais (ACP). A variabilidade dos dados foi explicada em $36,1 \%$, no eixo 1 , e $28,9 \%$ no eixo 2 , o que totalizou $65 \%$ da variabilidade total (Figura 2). O eixo 1 foi influenciado, principalmente, pelos grupos funcionais fitófago/praga e predador/parasita, que apresentaram autovetores positivos; enquanto o eixo 2 foi influenciado pelos grupos geófago/bioturbador e detritívoro/ decompositor, com autovetores positivos e negativos, respectivamente. No diagrama de ordenação, é possível analisar também as relações entre locais amostrados e os grupos funcionais. Observou-se uma tendência de

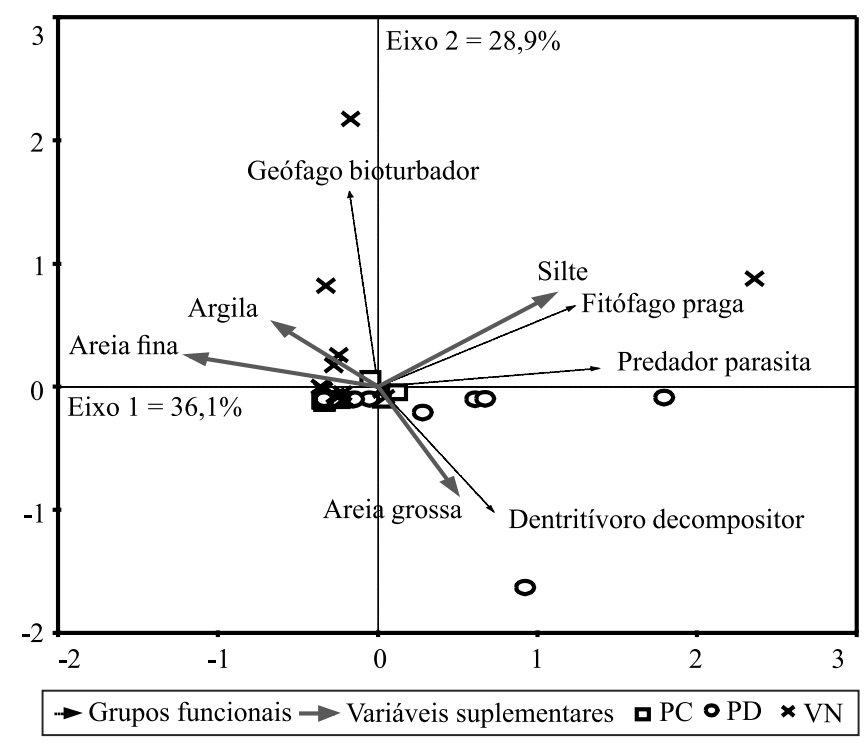

Figura 2. Análise de componentes principais para os grupos funcionais da macrofauna edáfica nos diferentes sistemas de uso e manejo avaliados, tendo-se utilizado as frações granulométricas dos solos como variáveis ambientais explicativas, no Sudoeste do estado do Piauí, na região de Matopiba.. PC, plantio convencional; PD, plantio direto; VN, vegetação nativa de cerrado. agrupamento dos pontos (locais) amostrados de acordo com os grupos funcionais, em que pontos nas áreas de VN estiveram mais associados aos grupos geófago/ bioturbador, fitófago/praga e predador/parasita. Por outro lado, as áreas sob PD apresentaram tendência de associação com o grupo detritívoro/decompositor.

Ao plotar a textura como variável explicativa no diagrama de ordenação, em razão de sua relação com os grupos funcionais, evidenciou-se que os grupos fitófago/praga e predador/parasita estão associados à fração silte do solo, e que o grupo detritívoro/ decompositor, com a de areia grossa. Já o grupo funcional geófago/bioturbador apresentou tendência de associação com as frações areia fina e argila. Segundo Moço (2006), a textura do solo é importante na determinação da disponibilidade de nutrientes para o sistema solo-planta, além de também ser determinante para a capacidade de retenção de água no solo.

Finalmente, ao se comparar o número de famílias encontrado em cada uma das áreas, observou-se que, sob PD, o número de famílias foi semelhante ao observado na área de referência. Ainda que a estrutura das comunidades varie entre as áreas, nota-se que o PD é um sistema mais equilibrado que o convencional, que proporciona melhor qualidade e quantidade de alimento para a macrofauna edáfica, com possíveis reflexos positivos sobre a manutenção da qualidade e do funcionamento do solo (Aquino \& Assis, 2007).

\section{Conclusões}

1. O cultivo do solo com sistemas de produção de grãos, em plantio direto ou convencional, altera a estrutura da comunidade de macrofauna edáfica, em comparação à condição natural de Cerrado.

2. O plantio direto proporciona maior abundância e riqueza de famílias que o preparo convencional do solo, e pode, portanto, ser recomendado como um sistema de produção conservacionista, que minimiza o impacto da atividade agrícola sobre a biodiversidade da macrofauna edáfica.

3. Os grupos Isoptera, Hymenoptera e Coleoptera são os mais abundantes nas áreas avaliadas, e a ordem Coleoptera apresenta maior número de famílias.

4. Os grupos funcionais geófago/bioturbador, fitófago/praga e predador/parasita estão associados a solos de textura argilo-siltosa, enquanto o grupo detritívoro/decompositor, a solos mais arenosos.

Pesq. agropec. bras., Brasília, v.51, n.9, p.1466-1475, set. 2016 DOI: 10.1590/S0100-204X2016000900045 


\section{Referências}

ABREU, R.R.L.; LIMA, S.S. de; OLIVEIRA, N.C.R. de; LEITE, L.F.C. Fauna edáfica sob diferentes níveis de palhada em cultivo de cana-de-açúcar. Pesquisa Agropecuária Tropical, v.44, p.409-416, 2014. DOI: 10.1590/S1983-40632014000400002.

ACOMPANHAMENTO DA SAFRA BRASILEIRA [DE] GRÃOS: safra 2014/15: sétimo levantamento, Brasília, DF, v.2, n.12, 2015. Disponível em: <http://www.conab.gov.br/OlalaCMS/ uploads/arquivos/15_04_10_09_22_05_boletim_graos_ abril_2015.pdf>. Acesso em: 2 mar. 2016.

ANDERSON, J.M.; INGRAM, J.S.I. Tropical soil biology and fertility: a handbook of methods. $2^{\text {nd }}$ ed. Wallingford: CAB International, 1993. 171p.

AQUINO, A.M. de; ASSIS, R.L. de. Agricultura orgânica em áreas urbanas e periurbanas com base na agroecologia. Ambiente e Sociedade, v.10, p.137-150, 2007. DOI: 10.1590/S1414753X2007000100009.

BARTZ, M.L.C.; PASINI, A.; BROWN, G.G. Earthworms as soil quality indicators in Brazilian no-tillage systems. Applied Soil Ecology, v.69, p.39-48, 2013. DOI: 10.1016/j.apsoil.2013.01.011.

BATISTA, I.; CORREIA, M.E.F.; PEREIRA, M.G.; BIELUCZYK, W.; SCHIAVO, J.A.; ROUWS, J.R.C. Frações oxidáveis do carbono orgânico total e macrofauna edáfica em sistema de integração lavoura-pecuária. Revista Brasileira de Ciência do Solo, v.38, p.797-809, 2014. DOI: 10.1590/S010006832014000300011.

BENITO, N.P.; BROSSARD, M.; PASINI, A.; GUIMARÃES, M. de F.; BOBILLIER, B. Transformations of soil macroinvertebrate populations after native vegetation conversion to pasture cultivation (Brazilian Cerrado). European Journal of Soil Biology, v.40, p.147-154, 2005. DOI: 10.1016/j.ejsobi.2005.02.002.

BROWN, G.G.; JAMES, S.W.; PASINI, A.; NUNES, D.H.; BENITO, N.P.; MARTINS, P.T.; SAUTTER, K.D. Exotic, peregrine, and invasive earthworms in Brazil: diversity, distribution, and effects on soils and plants. Caribbean Journal of Science, v.42, p.339-358, 2006.

BROWN, G.G.; NIVA, C.C.; ZAGATTO, M.R.G.; FERREIRA, S. de A.; NADOLNY, H.S.; CARDOSO, G.B.X.; SANTOS, A.; MARTINEZ, G. de A.; PASINI, A.; BARTZ, M.L.C.; SAUTTER, K.D.; THOMAZINI, M.J.; BARETTA, D.; SILVA, E. da; ANTONIOLLI, Z.I.; DECAËNS, T.; LAVELLE, P.M.; SOUSA, J.P.; CARVALHO, F. Biodiversidade da fauna do solo e sua contribuição para os serviços ambientais. In: PARRON, L.M.; GARCIA, J.R.; OLIVEIRA, E.B. de; BROWN, G.G.; PRADO, R.B. (Ed.). Serviços ambientais em sistemas agrícolas e florestais do Bioma Mata Atlântica. Brasília: Embrapa, 2015. p.121-154.

BRUYN, L.A.L. de. Ants as bioindicators of soil function in rural environments. Agriculture, Ecosystems and Environment, v.74, p.425-441, 1999. DOI: 10.1016/S0167-8809(99)00047-X.

CARRILLO, Y.; BALL, B.A.; BRADFORD, M.A.; JORDAN, C.F.; MOLINA, M. Soil fauna alter the effects of litter composition on nitrogen mineral soil. Soil Biology and Biochemistry, v.43, p.1440-1449, 2011. DOI: 10.1016/j.soilbio.2011.03.011.
CLAESSEN, M.E.C. (Org.). Manual de métodos de análise de solo. 2.ed. rev. e atual. Rio de Janeiro: Embrapa-CNPS, 1997. 212p.

CONSTANTINO, R. Padrões de diversidade e endemismo de térmitas no bioma Cerrado. In: SCARIOT, A.; SOUSA-SILVA, J.C.; FELFILI, J.M. (Org.). Cerrado: ecologia, biodiversidade e conservação. Brasília: Ministério do Meio Ambiente, 2005. p.319333.

CORREIA, M.E.F.; OLIVEIRA, L.C.M. de. Importância da fauna para a ciclagem de nutrientes. In: AQUINO, A.M. de; ASSIS, R.L. de (Ed.). Processos biológicos no sistema solo-planta: ferramentas para a agricultura sustentável. Brasília: Embrapa Informação Tecnológica; Seropédica: Embrapa Agrobiologia, 2005. p.77-99.

GILLER, K.E.; BEARE, M.H.; LAVELLE, P.; IZAC, A.-M.N.; SWIFT, M.J. Agricultural intensification, soil biodiversity and agroecossystem function. Applied Soil Ecology, v.6, p.3-16, 1997. DOI: 10.1016/S0929-1393(96)00149-7.

HANSKI, I.; CAMBEFORT, Y. Dung beetles ecology. Princeton: Princeton University, 1991. 481p. DOI: 10.1515/9781400862092.

HENDRIX, P.F.; PARMELEE R.W.; CROSSLEY JR, D.A.; COLEMAN, D.C.; ODUM, E.P.; GROFFMAN, P.M. Detritus food webs in conventional and no-tillage agroecosystems. BioScience, v.36, p.374-380, 1986. DOI: 10.2307/1310259.

HOUSE, G.J.; PARMELLE, R.W. Comparison of soil arthropods and earthworms from conventional and no-tillage agroecosystems. Soil and Tillage Research, v.5, p.351-360, 1985. DOI: 10.1016/ S0167-1987(85)80003-9.

LAVELLE, P. Assessing the abundance and role of invertebrate communities in tropical soils: aims and methods. Journal of African Zoology, v.102, p.275-283, 1988.

LAVELLE, P.; DECAËNS, T.; AUBERT, M.; BAROT, S.; BLOUIN, M.; BUREAU, F.; MARGERIE, P.; MORA, P.; ROSSIC, J.-P. Soil invertebrates and ecosystem services. European Journal of Soil Biology, v.42, p.3-15, 2006. Supplement 1. DOI: 10.1016/j.ejsobi.2006.10.002.

LAVELLE, P.; RODRÍGUEZ, N.; ARGUELLO, O.; BERNAL, J.; BOTERO, C. CHAPARRO, P.; GÓMEZ, Y.; GUTIÉRREZ, A.; HURTADO, M. del P.; LOAIZA, S.; PULLIDO, S.X.; RODRÍGUEZ, E.; SANABRIA, C.; VELÁSQUEZ, E.; FONTE, S.J. Soil ecosystem services and land use in the rapidly changing Orinoco River Basin of Colombia. Agriculture, Ecosystems and Environment, v.185, p.106-117, 2014. DOI: 10.1016/j. agee.2013.12.020.

LIMA, S.S. de; AQUINO, A.M. de; LEITE, L.F.C.; VELÁSQUEZ, E.; LAVELLE, P. Relação entre macrofauna edáfica e atributos químicos do solo em diferentes agroecossistemas. Pesquisa Agropecuária Brasileira, v.45, p.322-331, 2010. DOI: 10.1590/ S0100-204X2010000300013.

LOURENTE, E.R.P.; SILVA, R.F. da; SILVA, D.A. da; MARCHETTI, M.E.; MERCANTE, F.M. Macrofauna edáfica e sua interação com atributos químicos e físicos do solo sob diferentes sistemas de manejo. Acta Scientiarum. Agronomy, v.29, p.17-22, 2007. DOI: 10.4025/actasciagron.v29i1.60.

LUZ, R.A.; FONTES, L.S.; CARDOSO, S.R.S.; LIMA, É.F.B. Diversity of the Arthropod edaphic fauna in preserved and 
managed with pasture areas in Teresina-Piaui-Brazil. Brazilian Journal of Biology, v.73, p.483-489, 2013. DOI: 10.1590/S151969842013000300004.

MANHÃES, C.M.C.; GAMA-RODRIGUES, E.F.; MOÇO, M.K.S.; GAMA-RODRIGUES, A.C. Meso- and macrofauna in the soil and litter of leguminous trees in a degraded pasture in Brazil. Agroforestry Systems, v.87, p.993-1004, 2013. DOI: 10.1007/s10457-013-9614-0.

MARCHÃO, R.L.; LAVELLE, P.; CELINI, L.; BALBINO, L.C.; VILELA, L.; BECQUER, T. Soil macrofauna under integrated crop-livestock systems in a Brazilian Cerrado Ferralsol. Pesquisa Agropecuária Brasileira, v.44, p.1011-1020, 2009. DOI: 10.1590/ S0100-204X2009000800033.

MARCHÃO, R.L.; VILELA, L.; SANTOS JUNIOR, J. de D.G. dos; SÁ, M.A.C. de; BERGAMASCHI, L.C.; BORTONCELLO, L.R. Impactos de sistemas agrícolas nos atributos físicos, químicos e macrofauna num Latossolo do Oeste Baiano. Planaltina: Embrapa Cerrados, 2008. 30p. (Embrapa Cerrados. Boletim de pesquisa e desenvolvimento, 228).

MINGOTI, R.; BRASCO, M.A.; HOLLER, W.A.; LOVISI FILHO, E.; SPADOTTO, C.A. Matopiba: caracterização das áreas com grande produção de culturas anuais. Campinas: Embrapa Gestão Territorial, 2014. 2p. Nota técnica.

MOÇO, M.K. da S. Fauna do solo em diferentes agrossistemas de cacau no Sul da Bahia. 2006. 74p. Dissertação (Mestrado) - Universidade Estadual do Norte Fluminense Darcy Ribeiro, Campos dos Goytacazes.

NUNES, L.A.P.L.; SILVA, D.I.B. da; ARAÚJO, A.S.F. de; LEITE, L.F.C.; CORREIA, M.E.F. Caracterização da fauna edáfica em sistemas de manejo para produção de forragens no Estado do Piauí. Revista Ciência Agronômica, v.43, p.30-37, 2012.

OLIVEIRA, C.M. de. Aspectos bioecológicos do Coró-dashortaliças (Aegopsis bolboceridus) (Thomson) (Coleoptera: Melolonthidae) no cerrado do Brasil Central. Planaltina: Embrapa Cerrados, 2005. 28p. (Embrapa Cerrados. Documentos, 143).

OLIVEIRA, M.I.L. de; BRUNET, D.; MITJA, D.; CARDOSO, W. dos S.; BENITO, N.P.; GUIMARÃES, M. de F.; BROSSARD, M. Incidence of epigeal nest-building termites in Brachiariapastures in the Cerrado. Acta Scientiarum. Agronomy, v.33, p.181-185, 2011. DOI: 10.4025 /actasciagron.v33i1.7075.
PADOA, L. Effects of conservation agriculture on soil macrofauna and $\mathbf{C}$ and $\mathbf{N}$ levels in the Cerrado of Brazil. 2010. 21p. Dissertação (Mestrado) - Université Montpellier II, Faculté des Sciences, Montpellier.

PORTILHO, I.I.R.; CREPALDI, R.A.; BORGES, C.D.; SILVA, R.F. da; SALTON, J.C.; MERCANTE, F.M. Fauna invertebrada e atributos físicos e químicos do solo em sistemas de integração lavoura-pecuária. Pesquisa Agropecuária Brasileira, v.46, p.1310-1320, 2011. DOI: 10.1590/S0100-204X2011001000027.

PRAGANA, R.B.; RIBEIRO, M.R.; NÓBREGA, J.C.A.; RIBEIRO FILHO, M.R.; COSTA, J.A. da. Qualidade física de Latossolos Amarelos sob plantio direto na região do Cerrado piauiense. Revista Brasileira de Ciência do Solo, v.36, p.15911600, 2012. DOI: 10.1590/S0100-06832012000500023.

SANTOS, G.G.; SILVEIRA, P.M. da; MARCHÃO, R.L.; BECQUER, T.; BALBINO, L.C. Macrofauna edáfica associada a plantas de cobertura em plantio direto em um Latossolo Vermelho do Cerrado. Pesquisa Agropecuária Brasileira, v.43, p.115-122, 2008. DOI: 10.1590/S0100-204X2008000100015.

SILVA, R.F. da; AQUINO, A.M. de; MERCANTE, F.M.; GUIMARÃES, M. de F. Macrofauna invertebrada do solo sob diferentes sistemas de produção em Latossolo da região do Cerrado. Pesquisa Agropecuária Brasileira, v.41, p.697-704, 2006. DOI: 10.1590/S0100-204X2006000400022.

SOTHERTON, N.W. The distribution and abundance of predatory arthropods overwintering on farmland. Annals of Applied Biology, v.105, p.423-429, 1984. DOI: 10.1111/j.1744-7348.1984.tb03068.x.

SWIFT, M.J.; HEAL, O.W.; ANDERSON, J.M. Decomposition in terrestrial ecosystems. Oxford: Blackwell, 1979. 372p. (Studies in ecology, 5).

VENDRAME, P.R.S.; MARCHÃO, R.L.; BRITO, O.R.; GUIMARÃES, M.D.F.; BECQUER, T. Relationship between macrofauna, mineralogy and exchangeable calcium and magnesium in Cerrado Oxisols under pasture. Pesquisa Agropecuária Brasileira, v.44, p.996-1001, 2009. DOI: 10.1590/ S0100-204X2009000800031.

WINK, C.; GUEDES, J.V.C.; FAGUNDES, C.K.; ROVEDDER, A.P. Insetos edáficos como indicadores da qualidade ambiental. Revista de Ciências Agroveterinárias, v.4, p.60-71, 2005.

Recebido em 13 de agosto de 2015 e aprovado em 14 de abril de 2016

Pesq. agropec. bras., Brasília, v.51, n.9, p.1466-1475, set. 2016 DOI: 10.1590/S0100-204X2016000900045 